\title{
Parametric instability in oscillatory shear flows
}

\author{
By FRANCIS J. POULIN ${ }^{1} \dagger$, G. R. FLIERL ${ }^{2}$ AND J. PEDLOSK Y \\ ${ }^{1}$ Mathematical Institute, University of St. Andrews, St. Andrews, Fife, KY16 9SS, UK \\ ${ }^{2}$ Department of Earth, Atmospheric, and Planetary Sciences, \\ Massachusetts Institute of Technology, Cambridge, MA 02139, USA \\ ${ }^{3}$ Woods Hole Oceanographic Institution, MS 21, Woods Hole Road, Woods Hole, MA 02543, USA
}

(Received 19 September 2002 and in revised form 29 November 2002)

In this article we investigate time-periodic shear flows in the context of the twodimensional vorticity equation, which may be applied to describe certain large-scale atmospheric and oceanic flows. The linear stability analyses of both discrete and continuous profiles demonstrate that parametric instability can arise even in this simple model: the oscillations can stabilize (destabilize) an otherwise unstable (stable) shear flow, as in Mathieu's equation (Stoker 1950). Nonlinear simulations of the continuous oscillatory basic state support the predictions from linear theory and, in addition, illustrate the evolution of the instability process and thereby show the structure of the vortices that emerge. The discovery of parametric instability in this model suggests that this mechanism can occur in geophysical shear flows and provides an additional means through which turbulent mixing can be generated in large-scale flows.

\section{Introduction}

The Earth's atmosphere and oceans are forced by numerous different physical mechanisms that produce variability or periodicity on many different time scales, i.e. yearly, seasonally, daily and tidally. In addition, independently of any periodic forcings, oscillations can be generated from nonlinear dynamics in the form of amplitude vacillations in otherwise steady flows (Pedlosky 1987). Even though periodic flows are ubiquitous in nature, the instability of time-dependent shear flows has received relatively little attention in comparison to that of time-independent ones. Instead of studying a time-dependent flow, the time average of the basic state has often been analysed in the belief that it adequately describes the full problem. However, important information is lost in this approximation which can result in both quantitative and qualitative differences between the predictions and the observations. The time dependence is important since it can destabilize (stabilize) a flow, which consequently alters the transport of heat, momentum and mass (Davis 1976). The importance of time dependence has been emphasized in the context of internal waves in Broutman, Macaskill \& McIntyre (1997). In the present article, we study simple oscillatory shear flows in order to determine which phenomena can arise that are absent in the steady case.

$\dagger$ Present address: Mathematical Institute, North Haugh, Room 221, University of St. Andrews, St. Andrews, Fife, KY16 9SS, UK. 
Our definition of instability for a time-periodic basic state is taken from Rosenblat (1968) and Davis (1976). A periodic basic state is unstable if there exists a perturbation that experiences net growth over each period. If the perturbations decay at every instant, the basic state is said to be stable or monotonically stable. The system is transiently stable if there is no net growth after one cycle; however, there may be intervals when disturbances grow and other times when they decay.

A simple example of an oscillatory dynamical system is the pendulum with the suspension point oscillated vertically. Its linear stability is governed by Mathieu's equation. The stability of the stationary states can be altered by oscillating the suspension point (Stoker 1950); this change of stability is called parametric resonance or parametric instability.

Early experiments by Faraday using a square tank oscillated in the vertical direction proved that parametric instability can arise in the context of surface gravity waves (Benjamin \& Ursell 1954; Drazin \& Reid 1995). Subsequent studies have discovered that parametric instability can occur in internal gravity waves via a resonant wave triad interaction (McEwan \& Robinson 1975; Staquet \& Sommeria 2002). In this resonant triad, the energy is transferred from the two larger waves to the smallest one. Also, since the most unstable mode is a subharmonic, the instability transfers energy to longer time scales.

The first discovery of parametric instability in oscillatory shears was by Greenspan \& Benney (1963). They investigated a simple piecewise-linear velocity profile in the context of the barotropic two-dimensional vorticity equation. They determined that the periodic contractions and expansions of the jet generated parametric resonance. Our work differs significantly from Greenspan \& Benney (1963) in that we do not allow for the expansion and contraction in the fluid. Instead, we study parametric instability that arises in an incompressible flow with an oscillatory flow that has a non-zero mean shear.

The oscillatory Kelvin-Helmholtz problem was analysed by Kelly (1965) who discovered instances where the oscillations stabilized an otherwise unstable shear flow. However, he did not find examples where the oscillations destabilized the flow. Kelly's model is fundamentally different from ours in that his is irrotational (except at the interface) and the flow is stabilized by gravity and surface tension.

In order to determine for which wave amplitudes the secondary instabilities are dominant over the primary ones, a subsequent paper, Kelly (1967), studied the interaction of a steady shear with the oscillatory most unstable wave generated by the primary Kelvin-Helmholtz instability. This differs from our work in that the oscillatory state is non-parallel, since it has variation in the along-flow direction. Moreover, Kelly (1967) only finds instances where the oscillations destabilize the flow, with no evidence of stabilization.

Rosenblat (1968) analysed the stability of time-periodic azimuthal flows between coaxial, circular cylinders, which are typically referred to as modulated TaylorCouette flows. The physics of this problem is fundamentally different from the one we investigate since Taylor-Couette flow is a centrifugal instability in a curved geometry. In particular, Rosenblat (1968) considered perturbations in the vertical plane and assumed that they were azimuthally invariant. This implies that the instabilities that arise are associated with a convective-like overturning rather than the horizontal roll-up which occurs when waves on vorticity gradients are predominant.

Rosenblat's study of the inviscid dynamics with axisymmetric disturbances discovered several interesting properties arising from the oscillations of the basic state. First, the so-called rigid-body oscillations, where the azimuthal velocity is a function 
of the radial coordinate multiplied by a periodic function of time with zero mean, are all transiently stable. Second, a superposition of two different profiles that oscillate out of phase, each having a zero time average, generates parametric instability. Third, the linear stability problem of flows with non-zero mean flow and oscillations of infinitesimal amplitude is described by Mathieu's equation and therefore produces subharmonic resonance.

Since Rosenblat (1968) there have been theoretical and experimental studies of Taylor-Couette flow with viscosity for particular shear profiles (Walsh \& Donnelly 1988; Hu 1995; Ern \& Wesfreid 1999; Normand 2000; Lopez \& Marques 2002) which support the conclusion of Rosenblat (1968) that the oscillations can either stabilize or destabilize the flow. There have been other related studies of Taylor-Couette flows where each cylinder rotates azimuthally at a fixed rate but the inner cylinder oscillates in the radial direction (Marques \& Lopez 1997, 2000; Meseguer \& Marques 2000). They conclude that the oscillation in the axial direction always has a stabilizing effect. The study of oscillatory Poiseuille flow by Kerczek (1982) determined that oscillations either slightly stabilize or destabilize the flow, but there was no evidence of bifurcations in the stability properties.

Recently, Pedlosky \& Thomson (2002) studied the baroclinic instability of a zonal current on a beta-plane when the vertical shear is a periodic function of time. They discovered examples of parametric instability that would stabilize or destabilize the shear, but the instability mechanism is different from the one we study here.

In $\S 2$ we derive the equations that govern the linear stability of an oscillatory, horizontal, planar shear flow. Then in $\S 3$ we investigate the stability properties of pure oscillatory shear flow to show that if the temporal average is zero, the flow is transiently stable. Subsequently, in $\S 4$, we examine the interaction of a steady background flow of constant non-zero vorticity with an oscillatory shear having two jumps in vorticity. This simple example demonstrates that oscillations can either stabilize or destabilize the flow. We derive criteria to calculate the transition wavenumbers and then perform a multiple-scale analysis to determine the growth rate in the first subharmonic tongues in parameter space. We also study the mixed barotropic-parametric instability to discern what effect oscillations have on a barotropically unstable mode. Next, in $\S 5$ we solve the linear stability problem for continuous oscillatory shears, find examples of parametric instability, and study the nonlinear evolution of these flows. Finally, $\S 6$ contains a summary of our findings and a discussion of their importance to geophysical flows.

\section{Governing equations}

Consider the homogeneous two-dimensional Euler equations for a fluid of uniform depth. The incompressibility of the system permits the horizontal velocity field to be written in terms of a stream function, $\boldsymbol{u}=\hat{\boldsymbol{k}} \times \nabla \psi$. This implies that the vorticity $q=\hat{\boldsymbol{k}} \cdot \nabla \times \boldsymbol{u}$ and the stream function are related by

$$
q=\nabla^{2} \psi
$$

The two-dimensional vorticity equation is

$$
\frac{\partial q}{\partial t}+J(\psi, q)=F(y, t)
$$

where $J(A, B)=A_{x} B_{y}-A_{y} B_{x}$ is the Jacobian operator and subscripts denote partial differentiation. The along-flow coordinate, $x$, is periodic and the across-flow 
coordinate, $y$, is unbounded. The function $F(y, t)$ denotes the time-dependent forcing which maintains the oscillatory flow and defines the vorticity of the basic state $\bar{q}(y, t)$ by the equation

$$
\frac{\partial \bar{q}}{\partial t}=F(y, t) .
$$

To determine the stability of the basic state, we perturb the vorticity and stream function in the standard fashion:

$$
\begin{aligned}
q(x, y, t) & =\bar{q}(y, t)+q^{\prime}(x, y, t), \\
\psi(x, y, t) & =\bar{\psi}(y, t)+\psi^{\prime}(x, y, t) .
\end{aligned}
$$

The substitution of this into (2.1) implies that the perturbation vorticity is equal to the Laplacian of the perturbation stream function. To derive the equation that governs the linear perturbation dynamics, we substitute (2.4) and (2.5) into (2.2) and linearize to obtain

$$
\frac{\partial q^{\prime}}{\partial t}+\bar{u} \frac{\partial q^{\prime}}{\partial x}+\bar{q}_{y} \frac{\partial \psi^{\prime}}{\partial x}=0
$$

The fields $\bar{u}(y, t)$ and $\bar{q}_{y}(y, t)$ are the velocity and across-flow gradient of vorticity of the background flow.

The fact that our geometry is periodic in the $x$-direction enables us to decompose the perturbation fields as normal modes in $x$ with wavenumber $k$ such that both $q^{\prime}(x, y, t)$ and $\psi^{\prime}(x, y, t)$ are proportional to $\exp (\mathrm{i} k x)$. This simplifies the inversion of equation (2.1), yielding the following equation for the stream function in terms of the vorticity:

$$
\psi^{\prime}(x, y, t)=-\frac{1}{2 k} \int_{-\infty}^{+\infty} \exp \left(-k\left|y^{\prime}-y\right|\right) q^{\prime}\left(x, y^{\prime}, t\right) \mathrm{d} y^{\prime} .
$$

When this, along with the modal decomposition, is substituted into (2.6) we obtain

$$
\frac{\partial q^{\prime}}{\partial t}+\mathrm{i} k \bar{u} q^{\prime}=\frac{\mathrm{i}}{2} \bar{q}_{y} \int_{-\infty}^{+\infty} \exp \left(-k\left|y^{\prime}-y\right|\right) q^{\prime}\left(x, y^{\prime}, t\right) \mathrm{d} y^{\prime} .
$$

\subsection{Discrete spectrum}

Rather than study this integral equation in generality, let us restrict our attention to forcing functions of the form

$$
F(y, t)=\frac{\mathrm{d} S}{\mathrm{~d} t} \sum_{n=1}^{N} \Delta_{n} \mathscr{H}\left(y-y_{n}\right),
$$

where $\mathscr{H}(y)$ is the Heaviside step function, $S(t) \Delta_{n}$ is the vorticity jump across the line $y_{n}$ at time $t$ and $S(t)$ is, in general, a periodic function of $t$. This particular forcing yields a system composed of $N+1$ strips of uniform vorticity with $N$ contour interfaces. This system of dynamical equations is equivalent to that which would arise if we calculated the linearized equations from a contour dynamical perspective with $N$ interfaces (Zabusky \& Overman 1983; Dritschel 1989; Pullin 1992; Silveira \& Flierl 2002). 
By substituting (2.9) into (2.3) and integrating, we solve for the vorticity field of the basic state and its spatial gradient:

$$
\bar{q}(y, t)=S(t) \sum_{n=1}^{N} \Delta_{n} \mathscr{H}\left(y-y_{n}\right)+q_{0}(y),
$$

and

$$
\bar{q}_{y}(y, t)=S(t) \sum_{n=1}^{N} \Delta_{n} \delta\left(y-y_{n}\right)+\frac{\mathrm{d} q_{0}}{\mathrm{~d} y}(y) .
$$

A non-zero function of integration, $q_{0}(y)$, introduces a non-oscillatory component in the flow; it is a control parameter for stability. Throughout, we restrict our attention to the case where $q_{0}$ is constant. Integration of equation (2.10) yields the velocity of the basic state,

$$
\bar{u}_{m} \equiv \frac{\bar{u}\left(y_{m}\right)}{S(t)}=-\frac{q_{0}}{S(t)} y_{m}-\sum_{n=1}^{N} \Delta_{n} \max \left\{\left(y_{m}-y_{n}\right), 0\right\} .
$$

We substitute (2.11) into (2.8) to obtain the governing equation for the linear stability problem for the case of $N$ contours and discrete perturbations only:

$$
\frac{\mathrm{d} q_{m}^{\prime}}{\mathrm{d} t}=-\mathrm{i} k S(t) \bar{u}_{m} q_{m}^{\prime}+\frac{\mathrm{i}}{2} S(t) \Delta_{m} \sum_{n=1}^{N} \exp \left(-k\left|y_{m}-y_{n}\right|\right) q_{n}^{\prime} .
$$

The discrete nature of equation (2.13) implies that this model will not capture instabilities arising from the continuous spectrum. In the steady case, such modes decay algebraically (Case 1960), and the instability arises from the modes associated with the change in sign of the vorticity gradient, which (2.13) captures adequately. This does not rule out the possibility that the continuous modes could play a role in the oscillating shear problem, but it does seem much less likely that a critical layer is significant given the changes in shape and amplitude of the mean flow.

In the next section we set $q_{0}=0$, in which case all fields are directly proportional to a periodic function $S(t)$ : this gives us pure oscillatory flows. Then, in $\S 4$, we study a flow that comprises a superposition of an oscillatory jet and a non-zero mean shear: mixed oscillatory flows. The parameter $q_{0}$ is taken to be constant for the sake of mathematical simplicity. This is a good approximation for those situations where the oscillatory shear has a smaller length scale than the mean shear. We do not address other expressions for $q_{0}$ in this work but note that they are also of interest to study.

Throughout, we focus on the simple case where the basic state oscillates at only one forcing frequency. For a particular resonance, if there is only one frequency that generates this instability, the inclusion of other frequencies would make a negligible contribution to the instability process. Therefore this approach of considering a solitary frequency still yields meaningful results, even in the case of multiple forcing frequencies.

\section{Pure oscillatory flows}

\subsection{Discrete problem}

For steady flows ( $S=$ constant) it is natural to decompose the perturbation in normal modes and then treat each mode separately:

$$
q_{m}^{\prime} \equiv q^{\prime}\left(x, y_{m}, t\right)=\operatorname{Re}\left\{\hat{q}_{m} \exp (\mathrm{i} k x+\mathrm{i} \sigma t)\right\},
$$


where $k$ and $\sigma$ are the wavenumber and frequency of the perturbation. For notational simplicity we define an $N$-vector, $\boldsymbol{q}$, which has $\hat{q}_{m}$ as the $m$ th component. If we substitute (3.1) into (2.13), we obtain the algebraic eigenvalue problem

$$
\sigma \boldsymbol{q}=S \boldsymbol{M} \boldsymbol{q},
$$

where the $(m, n)$-component of the $N \times N$ matrix $\boldsymbol{M}$ is

$$
-k \delta_{m n} \bar{u}_{m}+\frac{1}{2} \Delta_{m} \exp \left(-k\left|y_{m}-y_{n}\right|\right)
$$

and $\delta_{m n}$ is the Kronecker delta function. If the imaginary part of any of the eigenvalues of (3.2) is negative, then the state is barotropically unstable.

To analyse time-dependent basic flows, we rewrite (2.13) as a system of ordinary differential equations

$$
\frac{\mathrm{d} \boldsymbol{q}}{\mathrm{d} t}=\mathrm{i} S(t) \boldsymbol{M} \boldsymbol{q} .
$$

Here, $\boldsymbol{q}$ is the vector having $q_{m}^{\prime}$ as its elements. In the case where $q_{0}=0$, the matrix $\boldsymbol{M}$ is constant and therefore the exact solution is

$$
\boldsymbol{q}=\exp \left(\mathrm{i} M \int_{0}^{t} S(s) \mathrm{d} s\right) \boldsymbol{q}_{0} .
$$

Assuming that $\boldsymbol{M}$ is non-defective, we can rewrite it as $\boldsymbol{M}=\boldsymbol{X} \boldsymbol{D} \boldsymbol{X}^{-1}$, where $\boldsymbol{D}$ is a matrix with eigenvalues in the diagonal and $\boldsymbol{X}$ is the matrix of column eigenvectors. By substituting the eigenvalue decomposition into (3.5) and setting $S(t)=\delta+\epsilon \cos (\omega t)$, the solution becomes

$$
\boldsymbol{q}=\boldsymbol{X} \exp \left(\mathrm{i} t\left[\delta+\epsilon \frac{\sin (\omega t)}{\omega t}\right] \boldsymbol{D}\right) \boldsymbol{X}^{-1} \boldsymbol{q}_{0} .
$$

Throughout this article, there is no restriction on the magnitude of $\epsilon$ and $\delta$ except in the multiple-scales analysis, where it is explicitly required that $\epsilon \ll 1$.

Since $\boldsymbol{D}$ is diagonal, the exponential matrix above is simply a diagonal matrix with each entry of the form

$$
\exp \left(\mathrm{i} t\left[\delta+\epsilon \frac{\sin (\omega t)}{(\omega t)}\right] c_{n}\right)
$$

$n$ ranges between 1 and $N$ and $c_{n}$ is the $n$th eigenvalue of $\boldsymbol{M}$. Note that if $\epsilon=0$, (3.6) reduces to the stability problem for the steady-state solution.

Observe that if the mean of the oscillatory flow is zero $(\delta=0)$, the periodic shear is transiently stable: there are intervals of both growth and decay, but after an entire period there is no net growth. This result is analogous to that of Rosenblat (1968), which states that all rigid-body oscillations of the Taylor-Couette flow are transiently stable.

In the general case where $\delta$ and $\epsilon$ are both non-zero, we have a superposition of the previous two cases. For an unstable wavenumber, if $\delta$ is positive, the mode will continually grow as in the steady-state theory and hence the solution is unstable. Therefore the stability of the unsteady flow is entirely determined by the mean profile: if the latter, viewed as a steady flow, is unstable (stable) then the flow oscillating around that mean will likewise be unstable (stable).

\subsection{Continuous problem}

The linear stability results we have just proved do not depend on the number of contour interfaces chosen in the forcing function (2.9). The fact that these results hold 
with increasing $N$ suggests that they will also hold for continuous profiles. This is the next issue we investigate, but first we study the stability of continuous steady flows. The perturbation is decomposed as

$$
q^{\prime}(x, y, t)=\operatorname{Re}\{\hat{q}(y) \exp (\mathrm{i} k x+\mathrm{i} \sigma t)\} .
$$

If we substitute this into (2.8) and assume $S$ is constant, we obtain the following eigenvalue problem:

$$
\sigma \hat{q}(y)=S \mathscr{M}(\hat{q}(y))
$$

in terms of the integral operator $\mathscr{M}$ defined as

$$
\mathscr{M}(\hat{q}(y))=-k \bar{u} \hat{q}+\frac{\bar{q}_{y}}{2} \int_{-\infty}^{+\infty} \exp \left(-k\left|y-y^{\prime}\right|\right) \hat{q}\left(y^{\prime}\right) \mathrm{d} y^{\prime} .
$$

For time-dependent basic states we must decompose the perturbation as

$$
q^{\prime}(x, y, t)=\operatorname{Re}\{\hat{q}(y, t) \exp (\mathrm{i} k x)\} .
$$

The linear stability problem is then governed by the integral equation

$$
\frac{\partial \hat{q}}{\partial t}(y, t)=\text { i } S(t) \mathscr{M}(\hat{q}(y, t)),
$$

in terms of the operator $(3.10) ; \bar{q}_{y}$ is now interpreted as a partial derivative. Assuming that $\hat{q}$ is $\hat{q}(t)$ times a $y$ structure which is an eigenfunction enables us to replace $\mathscr{M}$ by the eigenvalue $\sigma$ and obtain

$$
\frac{\mathrm{d} \hat{q}}{\mathrm{~d} t}=\mathrm{i} S(t) \sigma \hat{q}
$$

This gives

$$
\hat{q}=q_{0} \exp \left[\mathrm{i} \sigma \int^{t} S(s) \mathrm{d} s\right],
$$

analogous to (3.5). If $S(t)$ is constant, we obtain the steady-state case. If $S(t)$ has zero mean, all modes are transiently stable. Moreover, if $\delta$ and $\epsilon$ are both non-zero we obtain instabilities at precisely the same wavenumbers that are unstable in the steady case; in addition, the growth rates match those for the mean profile. If the integral operator $\mathscr{M}$ is complete then this analysis implies that the oscillatory continuous case is unstable if and only if the mean profile is. Any discretization of the continuous system, even with many interfaces, yields the same results. Apparently a single profile multiplied by $S(t)$ does not have a rich enough structure to generate new types of instabilities. (Again, we note that the operator is often not complete; the effects of the continuous spectrum are not known.)

\section{Mixed oscillatory shears: two-contour example}

In this section, we study mixed oscillatory shears, i.e. an oscillatory shear superimposed upon a steady shear. The non-oscillatory component is a flow with a uniform non-zero shear and the oscillatory jet contains two jumps in vorticity. The two jumps are located at $y_{1,2}=\mp a$ and the vorticity jumps are $\Delta_{1,2}=\mp 1$. The velocities at the two interfaces are $\bar{u}_{1,2}=\mp\left(1-q_{0} / S(t)\right) a$. The governing linear equation is obtained by specializing (2.13) to this particular shear flow and defining $K=2 a k$ :

$$
\frac{\mathrm{d} \boldsymbol{q}}{\mathrm{d} t}=\frac{\mathrm{i}}{2} S\left[\begin{array}{cc}
K\left(1-q_{0} / S\right)-1 & -\exp (-K) \\
\exp (-K) & -K\left(1-q_{0} / S\right)+1
\end{array}\right] \boldsymbol{q} .
$$


Again, we start with the case where $S$ is constant so that

$$
q_{m}^{\prime}=\exp (\mathrm{i} \sigma t) \hat{q}_{m},
$$

which yields the following eigenvalue problem:

$$
\frac{4 \sigma^{2}}{S^{2}}=\left(K\left(1-q_{0} / S\right)-1\right)^{2}-\exp (-2 K)
$$

A criterion that is necessary and sufficient for the imaginary part of the frequency to be zero, and therefore for linear stability, is (Dritschel 1989)

$$
\frac{q_{0}}{S}<1
$$

If $q_{0}=1$, the steady state is only stable for $0 \leqslant S \leqslant 1$, where $S=\delta$ for $\epsilon=0$.

We analyse the stability of periodic shear flows using Floquet theory. The particular oscillatory state we study is that with $q_{0}=1, \delta=1 / 2, \epsilon=1 / 2$ and $\omega=1$. Snapshots of the basic state at every instant in time are stable by the steady criterion (4.4); therefore, any instabilities that are found must be parametric in nature. Next, we determine the location of the transition points. We then use a multiple-scale analysis to determine the growth rates of the first subharmonic, which is the most unstable region and hence the most important.

To determine the growth rates of the basic state for a particular wavenumber involves two steps. First, the column vectors of the identity matrix are taken to be initial conditions for the dynamical system (4.1), which is integrated numerically by one period. The particular method we use to integrate this system of ordinary differential equations is the fourth-order Runge-Kutta scheme implemented in Matlab. The resulting matrix, called the Floquet matrix, determines the stability of the system. If the Floquet matrix has an eigenvalue that has a positive real component the system is unstable. The real part of this eigenvalue gives the growth rate over one period; we divide this by the period to determine the growth rate per unit of time.

\subsection{Transition points}

We rewrite equation (4.1) as a scalar second-order equation,

$$
\frac{\mathrm{d}^{2} q^{\prime}}{\mathrm{d} t^{2}}-\frac{1}{S} \frac{\mathrm{d} S}{\mathrm{~d} t} \frac{\mathrm{d} q^{\prime}}{\mathrm{d} t}+\left(\frac{S^{2}}{4}\left[\left(K\left(1-\frac{q_{0}}{S}\right)-1\right)^{2}-\exp (-2 K)\right]^{2}+\frac{\mathrm{i}}{2} K q_{0} \frac{1}{S} \frac{\mathrm{d} S}{\mathrm{~d} t}\right) q^{\prime}=0
$$

This equation is satisfied at both interfaces and hence the subscript has been dropped. We explicitly state Mathieu's equation in order that the reader may appreciate the similarities and differences of the two problems:

$$
\frac{\mathrm{d}^{2} q}{\mathrm{~d} t^{2}}+(\delta+\epsilon \cos (\omega t)) q=0 .
$$

The Wronskian of equation (4.5) is

$$
W(t)=W(0) \exp \left(-\int_{0}^{t} \frac{1}{S} \frac{\mathrm{d} S}{\mathrm{~d} t}\right)=W(0) \frac{\delta+\epsilon}{\delta+\epsilon \cos (\omega t)} .
$$

$W(t)$ is a periodic function of period $T=2 \pi / \omega$. This differs from Mathieu's equation which has a constant Wronskian. However, a periodic Wronskian is sufficient to conclude, as in Mathieu's equation, that the transition solutions between stability and instability must be of period $T$ or $2 T$. This result is not explicitly stated in Stoker 


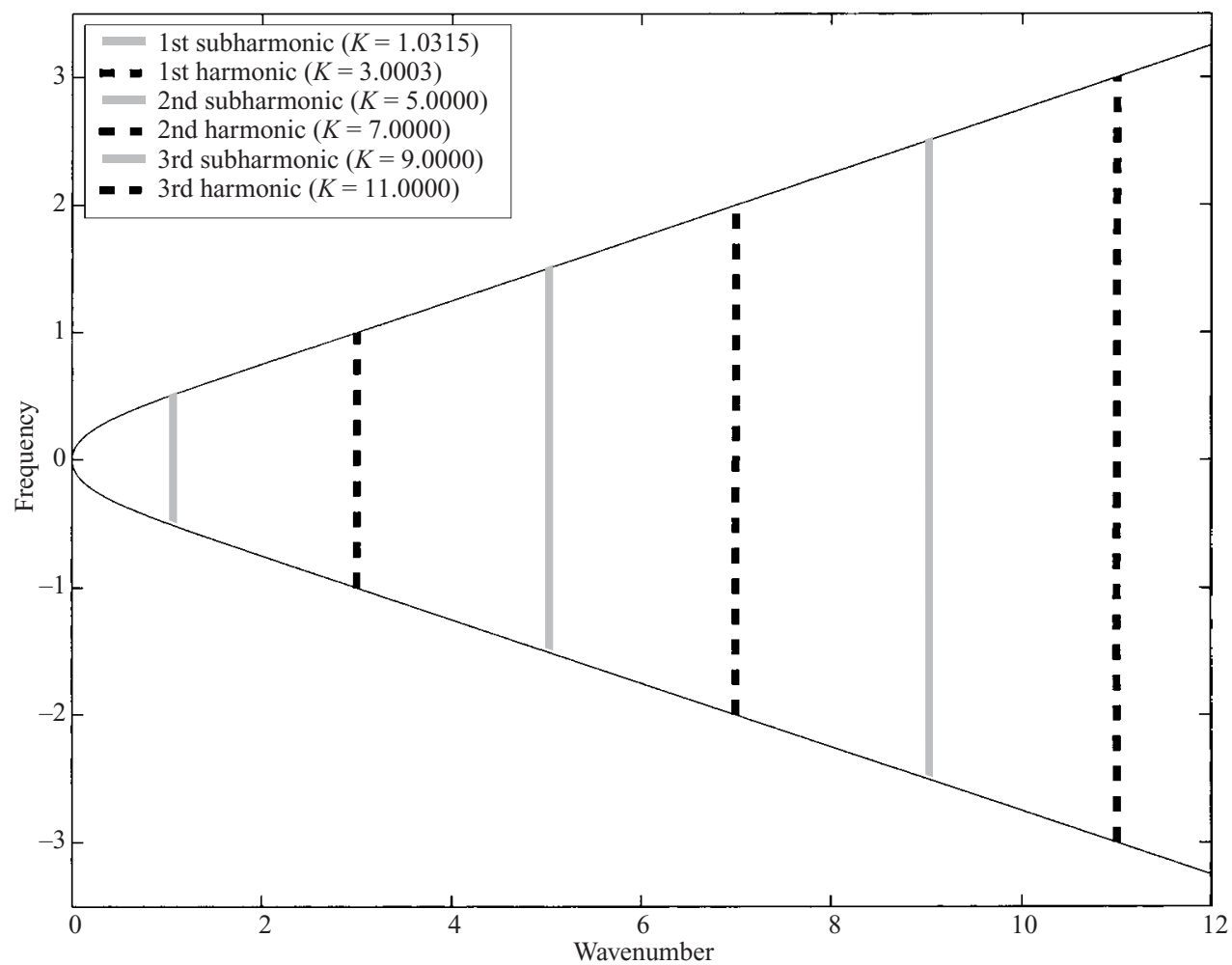

Figure 1 . The curved lines are the dispersion relations of the waves in the system with $S=1 / 2$, $q_{0}=1$ and $\omega=1$. The solid and dashed vertical lines indicate where the subharmonic and harmonic instabilities are located, respectively. These lines show the location of the transition wavenumbers, i.e. where a resonance occurs. The length of each is a multiple of the frequency, in this case 1 .

(1950), but it can be deduced from the discussion of "The Stability problem for Hill's equation and the Mathieu equation" in Chapter 6.

Consider the case of a nearly steady basic state, i.e. the asymptotic limit of $\epsilon \ll 1$ and $S \sim \delta$. The leading-order equation (4.5) reduces to the simple harmonic oscillator where the frequency $\sigma$ is defined by (4.3). Since the transition solutions must be of period $T$ or $2 T$, it is necessary that the frequency of the motion satisfy the relationship

$$
\sigma^{2}=\frac{n^{2} \omega^{2}}{4}
$$

where $n$ is a positive integer. This equation determines the position of the transition points in the case of $\epsilon=0$ and therefore where the regions of instabilities develop for small $\epsilon$; the odd and even $n$ correspond to subharmonics and harmonics, respectively. In particular, figure 1 illustrates the dispersion relationship for the waves in this two-contour problem. The vertical lines indicate the position of the subharmonic and harmonic transition points.

\subsection{Multiple scales}

Our next aim is to calculate analytically the growth rate of the first subharmonic in the regime $\epsilon \ll 1$ by using the method of multiple scales for arbitrary values of the 
two parameters $\delta / \omega$ and $q_{0} / \delta$. To begin, we define the matrices

$$
\boldsymbol{M}_{0}=\frac{\mathrm{i}}{2} \frac{\delta}{\omega}\left[\begin{array}{cc}
K\left(1-q_{0} / \delta\right)-1 & -\exp (-K) \\
\exp (-K) & -K\left(1-q_{0} / \delta\right)+1
\end{array}\right]
$$

and

$$
\boldsymbol{M}_{1}=\frac{\mathrm{i}}{2 \omega}\left[\begin{array}{cc}
K-1 & -\exp (-K) \\
\exp (-K) & -K+1
\end{array}\right]
$$

to rewrite the governing linear system (4.1) as

$$
\frac{\mathrm{d} \boldsymbol{q}}{\mathrm{d} t}=\boldsymbol{M}_{0} \boldsymbol{q}+\epsilon \cos (\omega t) \boldsymbol{M}_{1} \boldsymbol{q},
$$

where $\boldsymbol{q}$ is a time-dependent 2-vector. The fact that for Mathieu's equation the first subharmonic region grows linearly with the amplitude of the oscillations suggests that we should choose the long-time variable to be $T=\epsilon t$ (Stoker 1950).

If we expand $q$ as a perturbation series,

$$
\boldsymbol{q}=\boldsymbol{q}^{(0)}+\epsilon \boldsymbol{q}^{(1)}+O\left(\epsilon^{2}\right),
$$

and take the long-time variable to be $T=\epsilon t$, the leading-order equation is

$$
\frac{\partial \boldsymbol{q}^{(0)}}{\partial t}=\boldsymbol{M}_{0} \boldsymbol{q}^{(0)} \text {. }
$$

Since the vorticity is symmetric about $y=0$, the solution to our system is simply

$$
\boldsymbol{q}^{(0)}=S_{+}(T) \hat{\boldsymbol{s}}_{+} \exp (\mathrm{i} \sigma t)+S_{-}(T) \hat{\boldsymbol{s}}_{-} \exp (-\mathrm{i} \sigma t),
$$

where $\pm \mathrm{i} \sigma$ are the eigenvalues of $\boldsymbol{M}_{0}$ and $\hat{\boldsymbol{s}}_{ \pm}$are the right eigenvectors; that is,

$$
\boldsymbol{M}_{0} \hat{\boldsymbol{s}}_{ \pm}= \pm \mathrm{i} \sigma \hat{\boldsymbol{s}}_{ \pm} .
$$

Moreover, we define the adjoint problem

$$
\hat{\boldsymbol{s}}_{ \pm}^{\dagger} \boldsymbol{M}_{0}= \pm \mathrm{i} \sigma \hat{\boldsymbol{s}}_{ \pm}^{\dagger} .
$$

When $\sigma=\omega / 2$ we can let

$$
\boldsymbol{q}^{(1)}=\boldsymbol{q}_{+}(t) \exp (\mathrm{i} \sigma t)+\boldsymbol{q}_{-}(t) \exp (-\mathrm{i} \sigma t)+\boldsymbol{q}_{r}
$$

and the second-order equation then becomes

$$
\frac{\partial \boldsymbol{q}_{ \pm}}{\partial t}-\left[\boldsymbol{M}_{0} \mp \mathrm{i} \sigma\right] \boldsymbol{q}_{ \pm}+\frac{\mathrm{d} S_{ \pm}}{\mathrm{d} T} \hat{\boldsymbol{s}}_{ \pm}=\frac{1}{2} \boldsymbol{M}_{1} \hat{\boldsymbol{s}}_{\mp} S_{\mp} .
$$

Multiplying by the adjoints and requiring that the vectors $\boldsymbol{q}_{ \pm}$do not grow on the short time scale gives

$$
\left(\hat{\boldsymbol{s}}_{ \pm}^{\dagger} \hat{\boldsymbol{s}}_{ \pm}\right) \frac{\mathrm{d} S_{ \pm}}{\mathrm{d} T}=\frac{1}{2}\left(\hat{\boldsymbol{s}}_{ \pm}^{\dagger} \boldsymbol{M}_{1} \hat{\boldsymbol{s}}_{\mp}\right) S_{\mp} .
$$

These two equations are combined to yield two identical scalar second-order equations in $T$ :

$$
\frac{\mathrm{d}^{2} S_{ \pm}}{\mathrm{d} T^{2}}=\frac{1}{4} \frac{\left(\hat{\boldsymbol{s}}_{+}^{\dagger} \boldsymbol{M}_{1} \hat{\boldsymbol{s}}_{-}\right)\left(\hat{\boldsymbol{s}}_{-}^{\dagger} \boldsymbol{M}_{1} \hat{\boldsymbol{s}}_{+}\right)}{\left(\hat{\boldsymbol{s}}_{+}^{\dagger} \hat{\boldsymbol{s}}_{+}\right)\left(\hat{\boldsymbol{s}}_{-}^{\dagger} \hat{\boldsymbol{s}}_{-}\right)} S_{ \pm} .
$$

If the quantity in front of $S_{ \pm}$is positive, the basic state is unstable and the square root of this quantity gives the slope at which the growth rate increases in terms of $\epsilon$. 
The left and right eigenvectors are

$$
\hat{\boldsymbol{s}}_{ \pm}=\left[\begin{array}{c}
-\delta \exp (-K) \\
\pm \omega-K\left(\delta-q_{0}\right)+\delta
\end{array}\right], \quad \hat{\boldsymbol{s}}_{ \pm}^{\dagger}=\left[\begin{array}{c}
\delta \exp (-K) \\
\pm \omega-K\left(\delta-q_{0}\right)+\delta
\end{array}\right]^{T} .
$$

When we substitute these into (4.20), we conclude that the growth rate (in $T$ ) is

$$
\Sigma=\frac{\left|q_{0}\right| K \exp (-K)}{4 \omega^{2}},
$$

with the four parameters $K, q_{0}, \delta$ and $\omega$ related by

$$
\left(K\left(1-q_{0} / \delta\right)-1\right)^{2}-\exp (-2 K)=\frac{\omega^{2}}{\delta^{2}} .
$$

This equation is equivalent to (4.3) with $\sigma=\omega / 2$. If we consider the first three parameters to be independent and the frequency to be dependent, we can rewrite (4.22) in terms of the independent variables:

$$
\Sigma=\frac{\left|q_{0}\right| K \exp (-K)}{4 \delta^{2}\left[\left(K\left(1-q_{0} / \delta\right)-1\right)^{2}-\exp (-2 K)\right]} .
$$

The growth rate of the first subharmonic is equal to $\epsilon \Sigma$.

\subsection{Pure parametric instability}

To explore beyond the asymptotic limit of small-amplitude oscillations, we use Floquet theory and solve the problem numerically. Figure 2 shows the results from a series of calculations for various wavenumbers and forcing amplitudes. The solid lines are the numerical approximations to the transition curves for the first subharmonic, first harmonic and second subharmonics. These curves should extend down to the $K$-axis but do not because of the limited resolution in $K$ and small numerical error that arises in each integration. The dashed lines are contours of the net growth after one period with increments of $5 /(2 \pi) \times 10^{-2}, 5 /(2 \pi) \times 10^{-3}$ and $5 /(2 \pi) \times 10^{-4}$ for the lower three plots respectively. Our calculations indicate that for $\epsilon \leqslant 0.1$ the numerical solution of the Floquet problem is linear, as predicted from the multiple-scales analysis. Beyond this range, small quadratic deviations are present.

We tailor equation (4.3) to the specific parameters under consideration and obtain

$$
4 n^{2}=(K+1)^{2}-\exp (-2 K)
$$

The transition wavenumbers for $n=1,2,3$ are $K=1.0315, K=3.0003$ and $K=$ 5.0000. For larger $n$ the solutions are, to a very good approximation, $K=2 n-1$. The growth rate for the first subharmonic region, calculated from equation (4.22), is $0.091925 \epsilon$. The one calculated from the direct linear stability calculation is accurate to this many significant digits.

The instability tongues in figure 2 are similar to that of Mathieu's equation in several ways. First, the unstable regions originate from the transition wavenumbers, as predicted from the leading-order theory. Second, the transition curves for the first subharmonic are linear and those for the higher harmonics are quadratic or higher. Third, the growth rate increases with $\epsilon$ more slowly on moving to higher harmonics. By plotting slices of this figure for fixed values of $\epsilon$, we have observed that the growth 

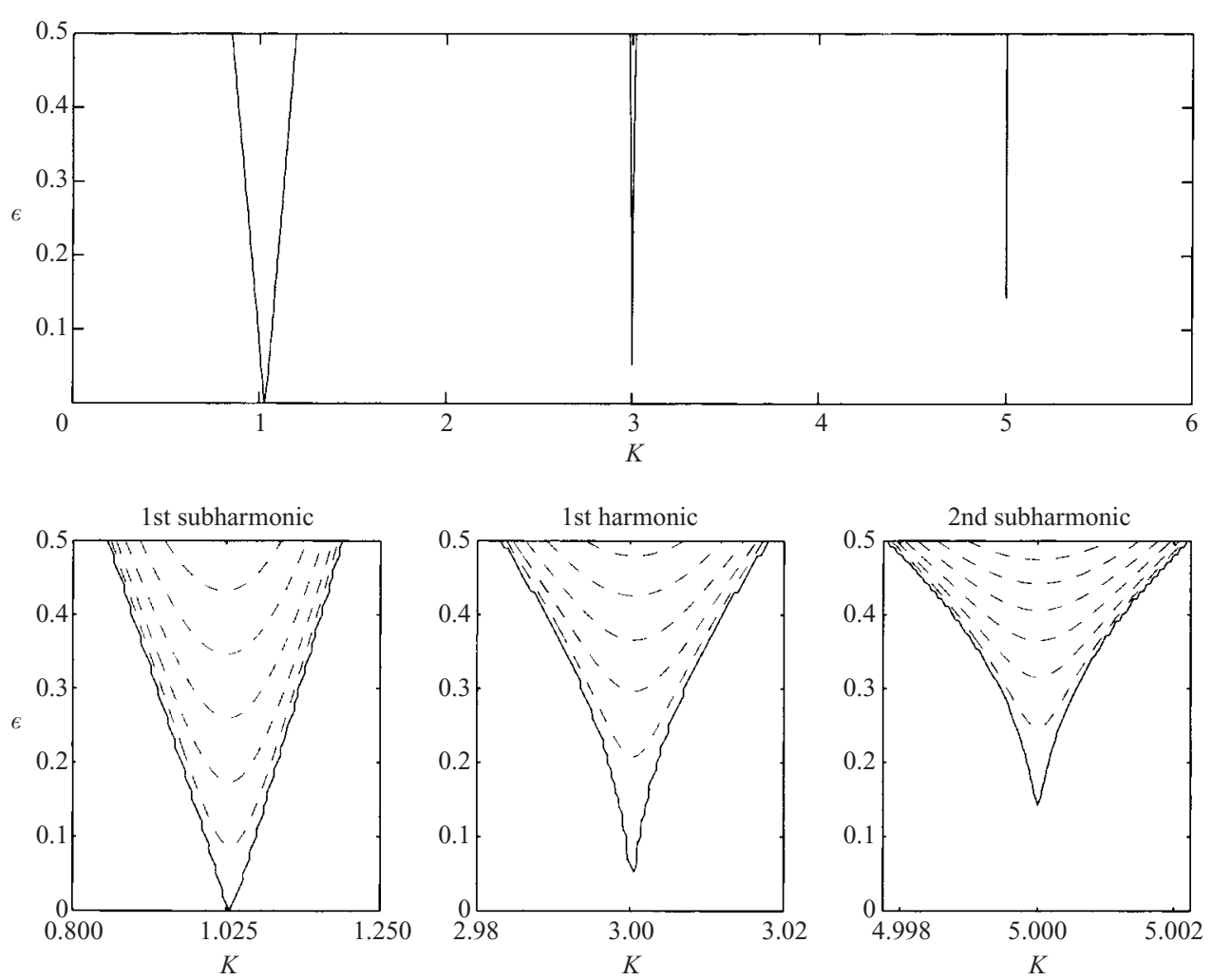

FIGURE 2. The upper plot depicts the stability boundaries of the first three unstable regions; first subharmonic, first harmonic and second subharmonic. The lower three focus on each of these modes and show the stability boundaries along with equally spaced growth rate contours. The dashed lines are contours of the growth rates in one period, with increments of $5 /(2 \pi) \times 10^{-2}, 5 /(2 \pi) \times 10^{-3}$ and $5 /(2 \pi) \times 10^{-4}$ for the lower three figures respectively.

rate decays exponentially with the wavenumbers, as predicted by equation (4.22). The growth rate decreases with wavenumber because the instability requires that the interfaces be in close proximity to each other (in comparison with the cross-stream decay scale for the waves) so that they can interact.

In figure 3, we plot the growth and phase of the disturbance for the wavenumber $K=1.02$ at $\epsilon=1 / 2$ throughout one period. In the top and bottom rows, we plot the modulus and phase of the growing and decaying modes, respectively. The solutions are obtained by numerically integrating the governing system for one period with the eigenvectors of the Floquet matrix as the initial conditions. Observe that the plots of the modulus have only one curve, as is true in general, since the perturbation vorticities at the two interfaces grow equally at all times. The majority of the growth (decay) occurs during the first and final quarters of the forcing period; this coincides with the intervals where $\cos (\omega t)$ is positive. We speculate that during these times the resonant triad, between the two waves and the basic state, can most effectively extract energy from the mean flow. The phases of the interfaces $y=-a$ and $y=a$ decrease and increase, respectively, by a factor of $\pi$ radians, as is required for it to be a first subharmonic. Clearly, there is a qualitative difference between pure and mixed oscillatory flows since our simple example of the latter produces parametric instability but the entire class of the former possesses none. 
Growing mode
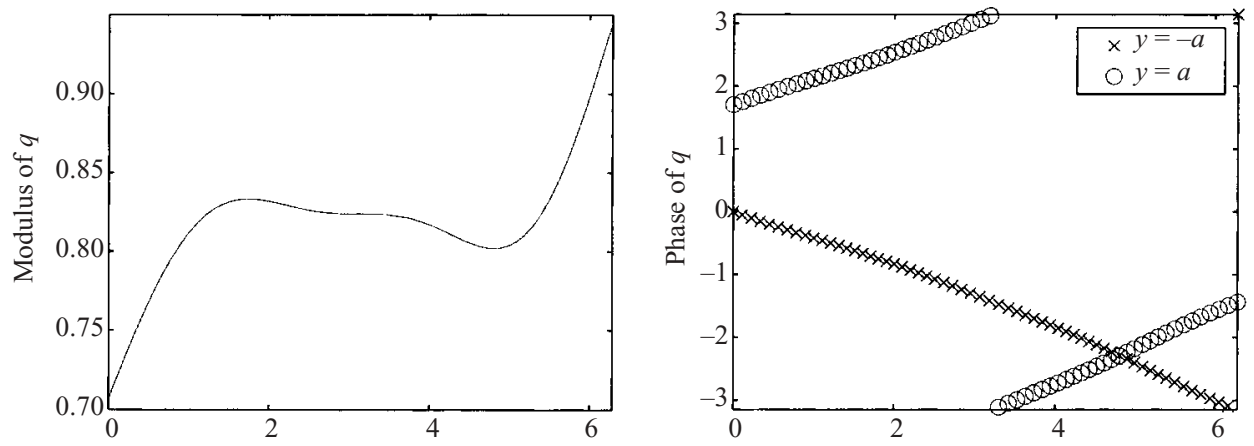

Decaying mode
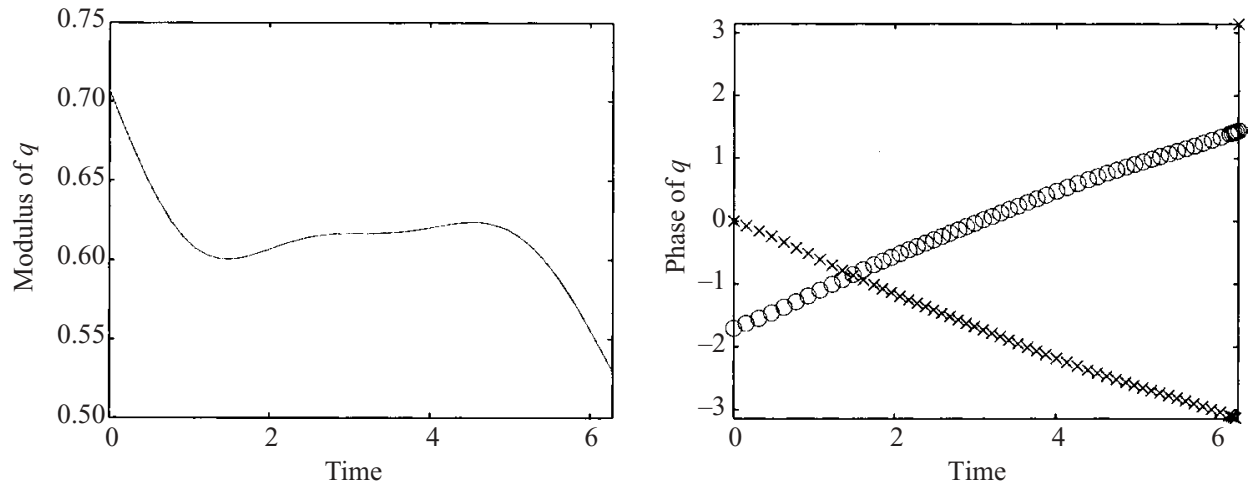

Figure 3. First subharmonic; $K=1.02, \epsilon=1 / 2$. The two plots in the left column show that the modulus of the growing and decaying modes have the same functional dependence on time. The phase plots show that the change in phase after one period is $\pi$, which indicates that this is the first subharmonic.

To derive a necessary condition for parametric resonance, consider (4.11) for the general case where the matrices $\boldsymbol{M}_{0}$ and $\boldsymbol{M}_{1}$ are arbitrary but constant. If they commute, as is true for pure oscillatory flow since $\boldsymbol{M}_{0}=\boldsymbol{M}_{1}$, the exact solution is

$$
\boldsymbol{q}=\exp \left(\boldsymbol{M}_{0} t+\epsilon \boldsymbol{M}_{1} \frac{\sin (\omega t)}{\omega}\right) \boldsymbol{q}_{0} .
$$

This solution does not give rise to parametric instability since the only instabilities are those that exist when $\epsilon=0$. Therefore, a necessary condition for parametric resonance is that $\boldsymbol{M}_{0}$ and $\boldsymbol{M}_{1}$ do not commute, as is the case with Mathieu's equation and with equation (4.1). In general, we expect that dynamical systems described by equation (4.11) will have matrices that do not commute so that parametric instability will be a common phenomenon in periodic flows.

Our example demonstrates that even though every snapshot in time is stable, and hence the average is certainly stable, the oscillatory flow may be unstable; the time dependence must be incorporated to properly determine the stability of the flow and the growth rates of disturbances. In modelling geophysical phenomena, one often uses the average flow as representative of the time-dependent flow. This may seem like a good approximation but it cannot account for instabilities that are inherent to the time-dependent nature of the flow. 

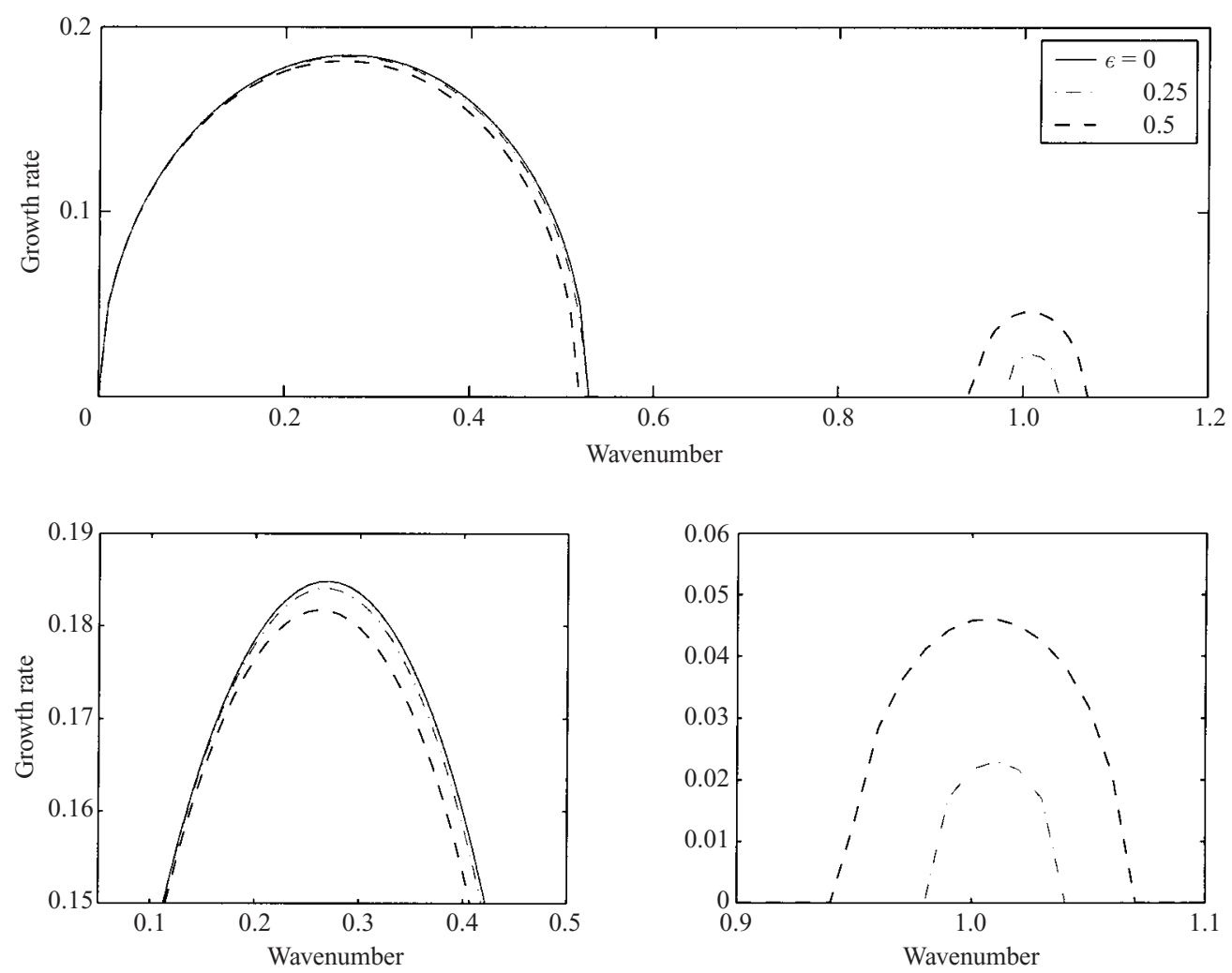

FIGURE 4. The transition from the purely barotropic instability to mixed barotropic-parametric instability. As $\epsilon$ increases, the growth rate of the barotropic mode gradually decreases and parametric modes are introduced and become more unstable.

\subsection{Mixed barotropic-parametric instability}

We now consider how barotropically unstable modes are affected by oscillatory shear through exploring the example of $q_{0}=-1, \delta=1 / 2$ and $\epsilon=0,1 / 4$, and $1 / 2$. A qualitative difference from the example in the previous subsection is that at every instant in time this case is barotropically unstable. The calculations of the growth rates within a period are presented in figure 4 . The top plot shows the growth rates for the three different $\epsilon$ and the bottom two focus in on the two separate modes: the barotropic and the parametric. As $\epsilon$ increases from 0 to $1 / 2$, the barotropic modes are stabilized: the maximum growth rate decreases and the range of the barotropic modes decreases. Moreover, as $\epsilon$ increases, parametric modes are introduced, their growth rates increase, and their range of unstable wavenumbers increases. This example illustrates how oscillations can either stabilize or destabilize waves, in comparison to the stability of the time-averaged state.

To predict the position of these transition points we specialize (4.3) to the case where $q_{0}=-1$ :

$$
4 n^{2}=(3 K-1)^{2}-\exp (-2 K) \text {. }
$$

We solve this nonlinear algebraic equation for $n=1,2,3$ and find that the transition wavenumbers are $K=1.0109, K=1.6681$ and $K=2.3336$, respectively. For larger $n$ the solutions are, to a very good approximation, $K=(2 n+1) / 3$. We apply the multiple-scale analysis and deduce that the growth rate of the first subharmonic is 
Growing mode
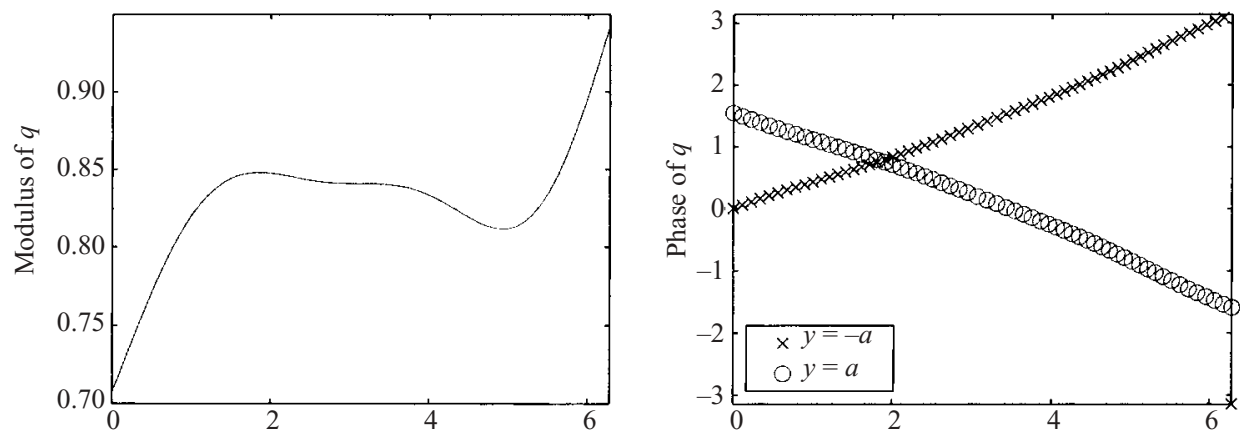

Decaying mode
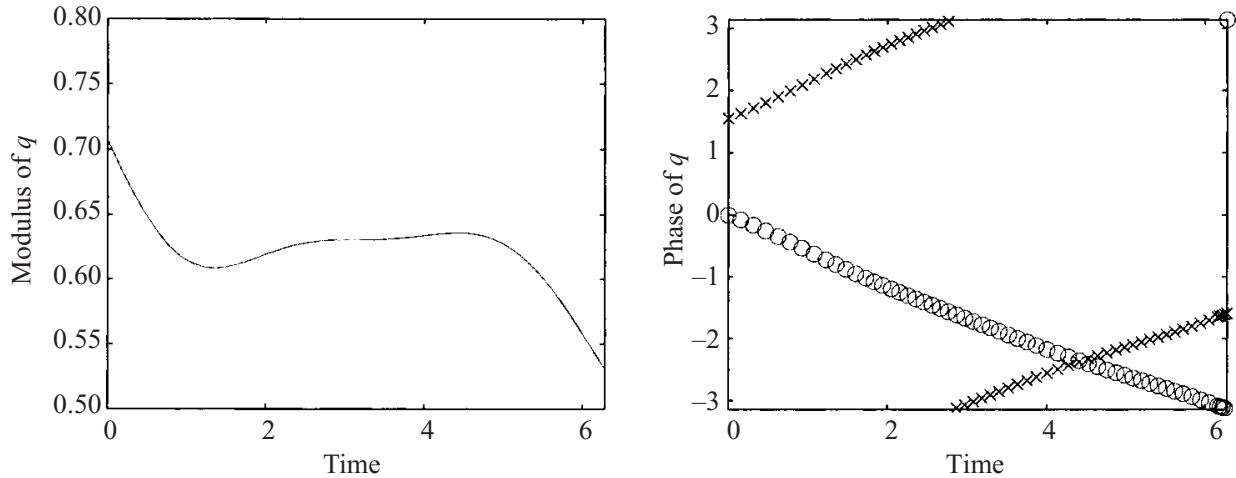

FIGURE 5. The first subharmonic mode in the case of mixed barotropic-parametric instability; $K=1, \epsilon=1 / 2$. It behaves qualitatively in the same fashion as the example of pure parametric instability.

$0.091965 \epsilon$. The linear coefficient in the growth rate calculated from directly solving the linear problem is accurate to within $10^{-6}$. The first subharmonic wavenumber from this example differs by only $10^{-2}$ from the one obtained in case of pure parametric instability. This causes a difference of $10^{-3}$ in the coefficient of the growth rate. In figure 5 , we present the growing and decaying modes for $\epsilon=1 / 2$ for the wavenumber $K=1$. The similarities of the modulus and phase between this figure and those in figure 3 imply that these first subharmonic modes are the same. These results suggest that the strength of the parametric instability is independent of whether the oscillatory flow is stable or unstable.

Figure 6 shows the results for the same values as before except that $\epsilon=1 / 10$. The phase plots are similar to before but the growth rates differ. Both the unstable and stable modes grow for the first half of the period and then decay for the second half; however, the amount of growth does not equal the amount of decay.

The final set of structural plots in figure 7 shows how the barotropic mode is altered by strong oscillations, in particular $\epsilon=1 / 2$. The growth plots are not exponential, as in the steady case, but resemble those of figure 3 . The phase plots, which are constant in the steady case, develop wiggles but the waves do not have a significant phase shift, if any, after one period. Therefore, the phase shift after one period gives a means for differentiating between the barotropic and parametric modes in the regime of strong oscillations. 
Growing mode
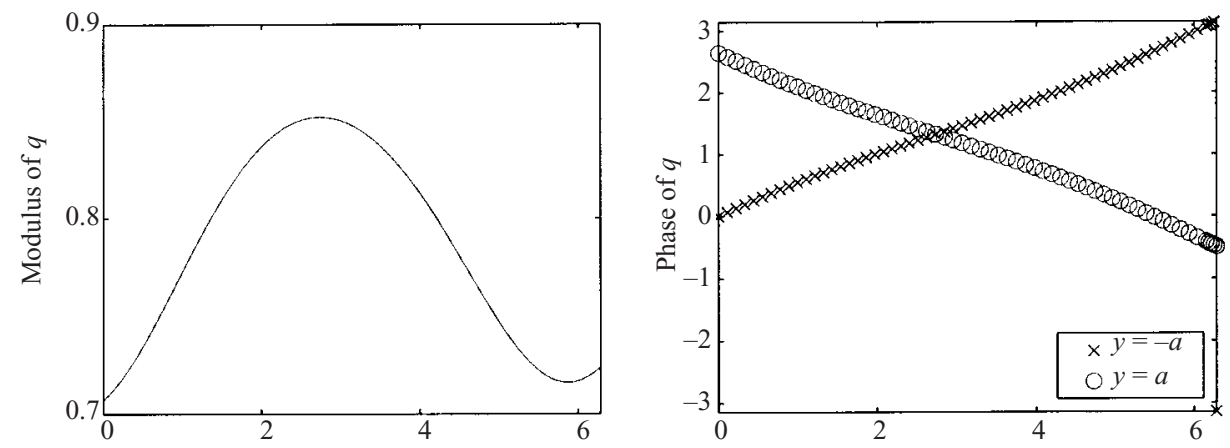

Decaying mode
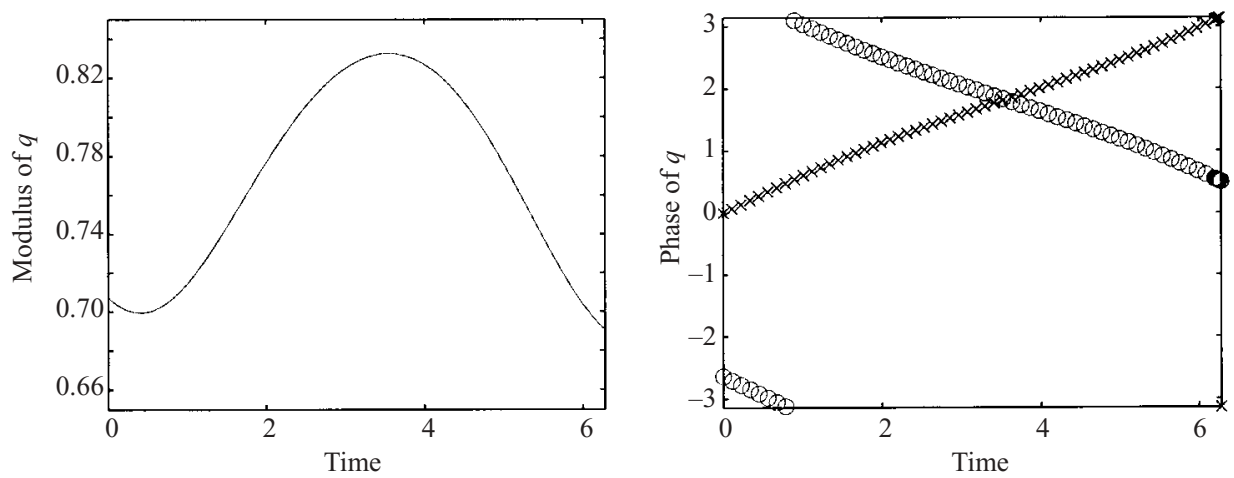

Figure 6. The first subharmonic mode for parametric instability with smaller forcing amplitude $(\epsilon=1 / 10)$ than in figure 5 . The result is that the growth is very slight but the phase difference is essentially the same.

\section{Continuous profiles}

We next study temporally periodic shear flows with continuous vorticity profiles. This work will demonstrate that parametric instability is not an artifact of the non-smoothness of the piecewise-linear velocity fields, but is indeed a real physical mechanism for instability. Our model is the same as before, a steady background shear superimposed upon an oscillatory jet, but now the oscillatory flow is taken to be continuous. In particular, we choose a family of Gaussian-like vorticity profiles of the form

$$
S(t) \exp \left(-(2 y)^{2 n}\right),
$$

where $n$ is a positive integer. In the limit as $n \rightarrow \infty$, they converge to the two-contour example studied in $\S 4$. For any finite value of $n$, the basic state is smooth. As $n$ increases, so does the steepness of the vorticity. Figure 8 illustrates the two-contour vorticity, the vorticity of several members of this family and the hyperbolic tangent shear layer profile, i.e. $\bar{q}=\operatorname{sech}^{2}(2 \mathrm{y})$.

\subsection{Linear stability analysis}

The linear stability analysis of the previous sections is extended to study this model with an arbitrary number of contours. The mean shear has vorticity $q_{0}$ and the oscillatory vorticity is given by equation (5.1) with $S(t)=\delta+\epsilon \cos (\omega t)$. In particular, 
Growing mode
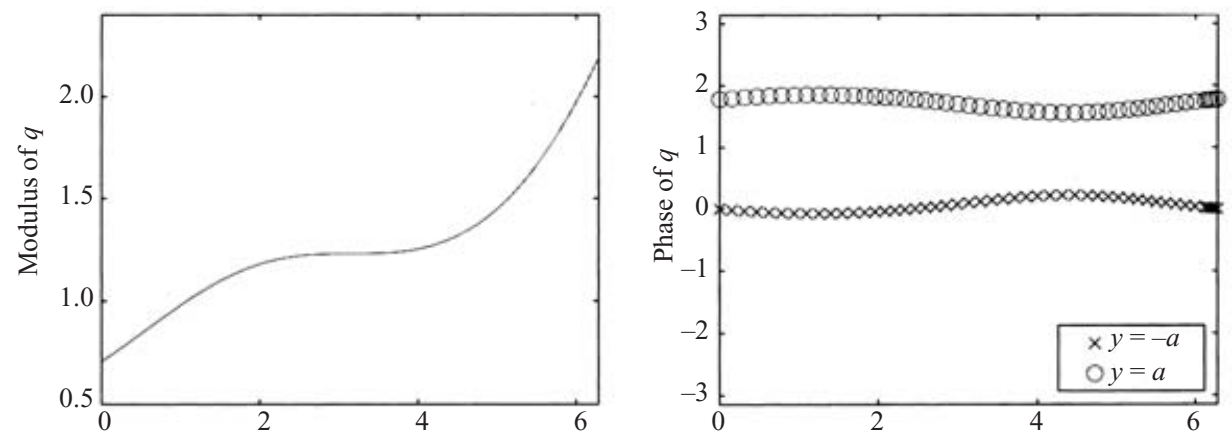

Decaying mode
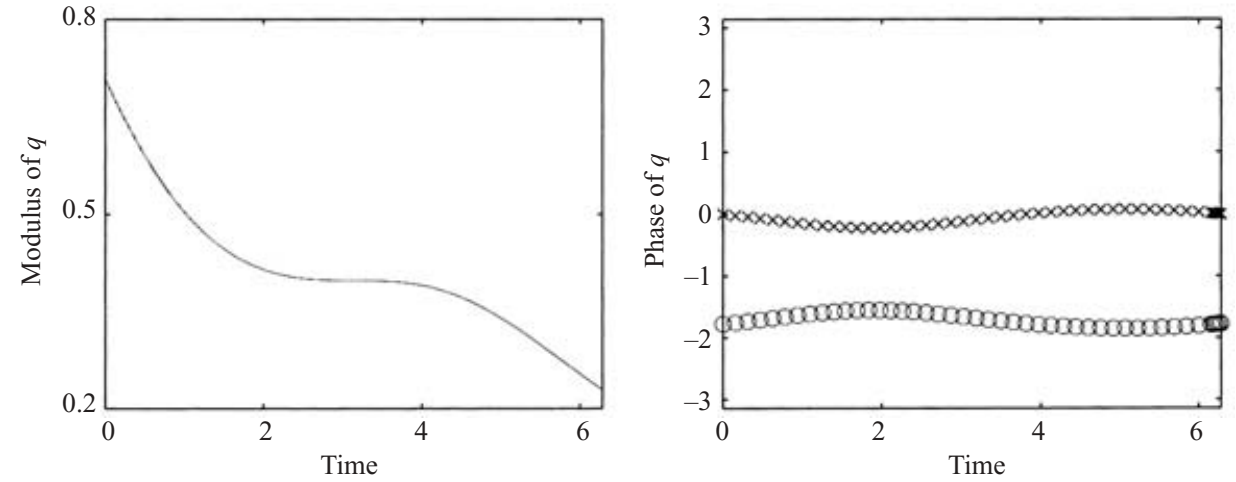

FIGURE 7. The barotropic mode altered by parametric resonance. The growth is not exponential, as in the steady case, but is similar to that of a parametric mode. There is also no net change in the phases of the interfaces.

we set $\delta=1 / 2=\epsilon$ and $\omega=1$. Fjortoft's theorem can be used to demonstrate that snapshots of these flows at every instant in time are stable. Therefore, any instabilities that arise in the oscillatory system are purely parametric in nature.

To solve the linear stability problem we discretized the shear flows into $N=200$ contours. By comparing calculations for different resolutions it was determined that for most of our profiles, the growth rates were predicted well by using as few as $N=50$ contours. The growth rates of parametric instabilities for the subharmonic region for the vorticity profiles of figure 8 are plotted in figure 9 . We have computed similar plots for other frequencies and we have observed qualitatively similar results.

Observe that increasing values of $n$ yield growth rates that approach the growth rate of the two-contour example. In particular, as $n$ decreases the instabilities are weakened and the most unstable modes occur at larger wavenumbers. We have not plotted the case of $n=1$ and the $\operatorname{sech}^{2}$ profile, since they are either stable or their growth rates are on the same order as the error; we have not found any evidence of instability for these gradual profiles.

Kelly (1965) discovered instances of parametric instability in the oscillatory KelvinHelmholtz problem by using a one-contour model. He mentions a continuous version of this problem with the one-contour profile replaced by a hyperbolic tangent profile. However, contrary to the discrete model, the continuous profile appeared to be stable. This led him to suggest that parametric instability would not be important 


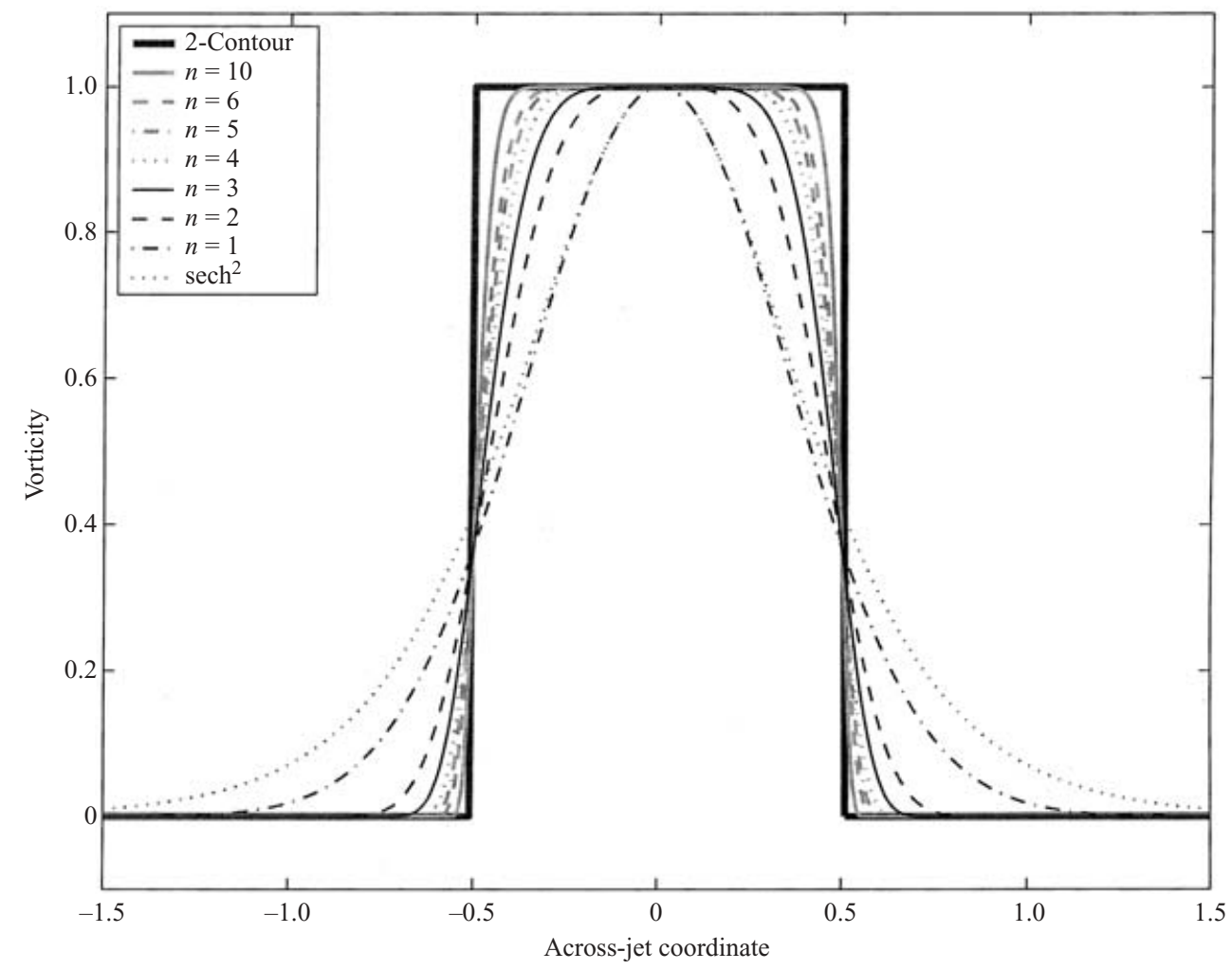

FIGURE 8 . The oscillatory components of the vorticity used: the two-contour example from $\S 4$ along with various Gaussian curves defined in (5.1) and the $\operatorname{sech}^{2}$ jet profile.

in continuous systems. We speculate that if instead he had replaced the one-contour profile with a velocity profile from our Gaussian family with $n \geqslant 2$, he would have discovered many examples of parametric instability in a continuous system. Indeed, this instability requires steep gradients in vorticity and the hyperbolic tangent profile in velocity is not steep enough. This is why the instabilities appear to be strongest in the one- and two-contour examples in Kelly (1965) and our work, respectively.

The two-contour example demonstrated that there is an infinite number of unstable modes, subharmonic or harmonic in nature, with amplitude decaying exponentially with wavenumber. The continuous flows also show a similar structure. By studying the first harmonic, we observed the tendency for the growth rates to decrease with decreasing $n$ and the instabilities to move to larger wavelengths. We surmize that the same qualities should apply to the higher harmonics; however, since their growth rates are very small, they are of little physical importance.

\subsection{Nonlinear simulations}

Our final objective in this section is to study the evolution of parametric instability in the nonlinear regime. To do so we have performed a series of numerical experiments that solve the nonlinear barotropic vorticity equation, defined by equations (2.2) and (2.1), with oscillatory forcing. The numerical method uses the third-order AdamsBashford finite-difference scheme to step the vorticity equation forward in time and a third-order upwind scheme for the advection on a $128 \times 128$ grid (Fletcher 1991). 


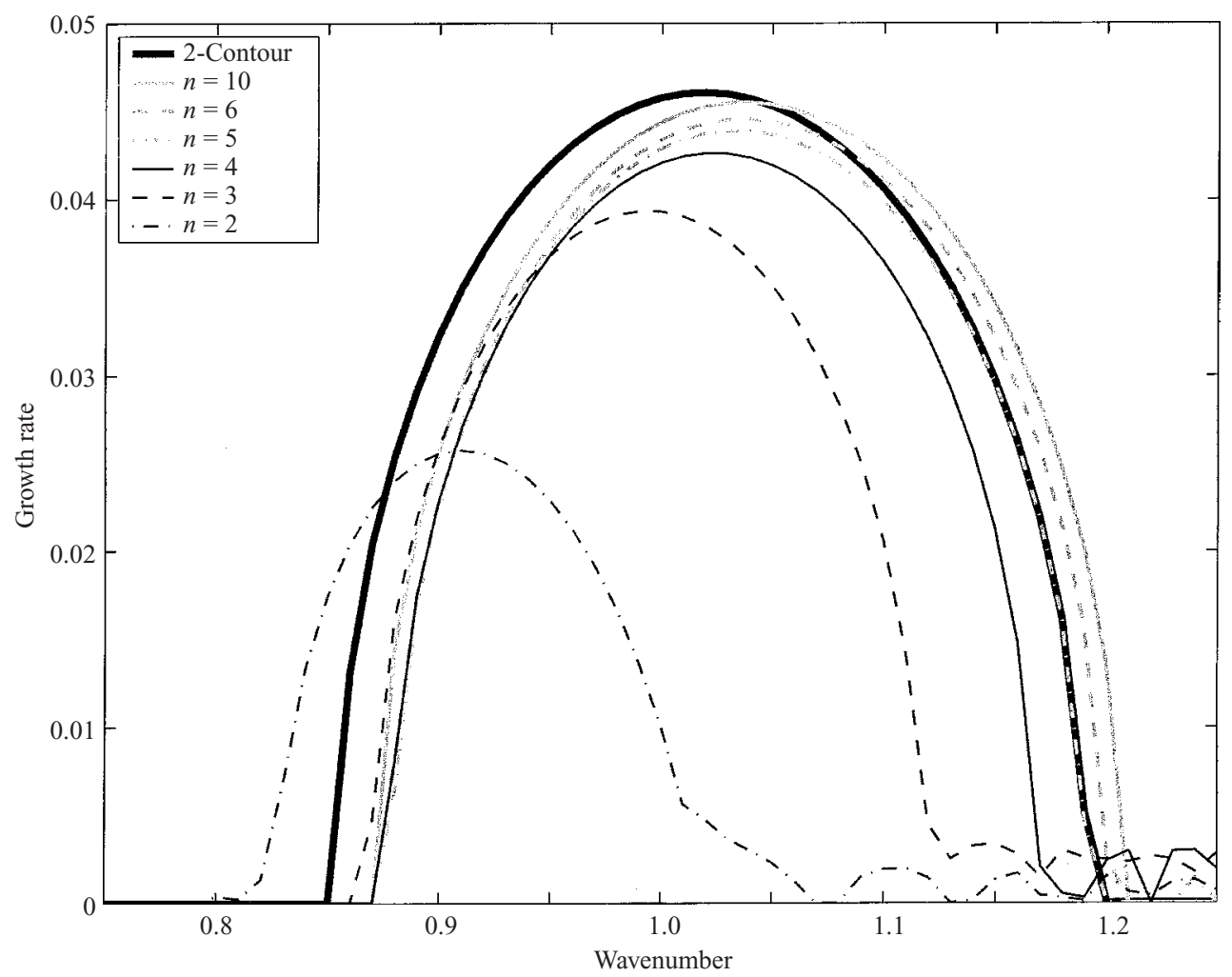

FiguRE 9. The growth rates of the first subharmonic region for various basic-state profiles with $\omega=1, \delta=1 / 2, \epsilon=1 / 2$ and $q_{0}=1$. Note that as $n$ decreases the growth rates decrease and the instabilities are shifted to larger wavelengths.

There is enough implicit diffusion contained in the advection scheme that no explicit diffusion was needed to stabilize the method. A spectral scheme is used to invert the vorticity in order to calculate the stream function. Calculations at different resolutions were performed to verify that the instabilities were robust and not numerical artifacts. To plot the solution at increments of the period, we have changed the frequency to $\omega=\pi / 4$, so that the period is $T=8$. The remaining parameters are as before, $\epsilon=1 / 2=\delta$ and $q_{0}=1$, and we study oscillatory vorticity profiles that are members of the Gaussian family (5.1). In particular, the forcing function is given by equation (2.3).

Figure 10 compares the growth rates calculated from the linear theory with those computed from the nonlinear simulations. We used monochromatic simulations but remark that with polychromatic simulations, which include the first six modes that fit in the domain, we obtained the same structures. The reason is that the geometry is chosen so that the most unstable mode fits exactly in the domain whereas the other first subharmonics do not and, therefore, cannot grow. Moreover, the harmonics and higher-order subharmonics are too weak to play a significant role during the stage of linear growth. Initially, the polychromatic simulations exhibit a blend of the initial modes. However, the wave $k=\pi$ eventually appears and then grows exponentially, surpassing all other structures in the system. The linear and nonlinear calculations of the growth rate vary by no more than $4 \%$.

The amplitude for $n=4$ is plotted in figure 11 in two semi-log plots; plot $(b)$ magnifies a region in $(a)$. In the interval from $t=40$ to $t=150$, the envelope of 


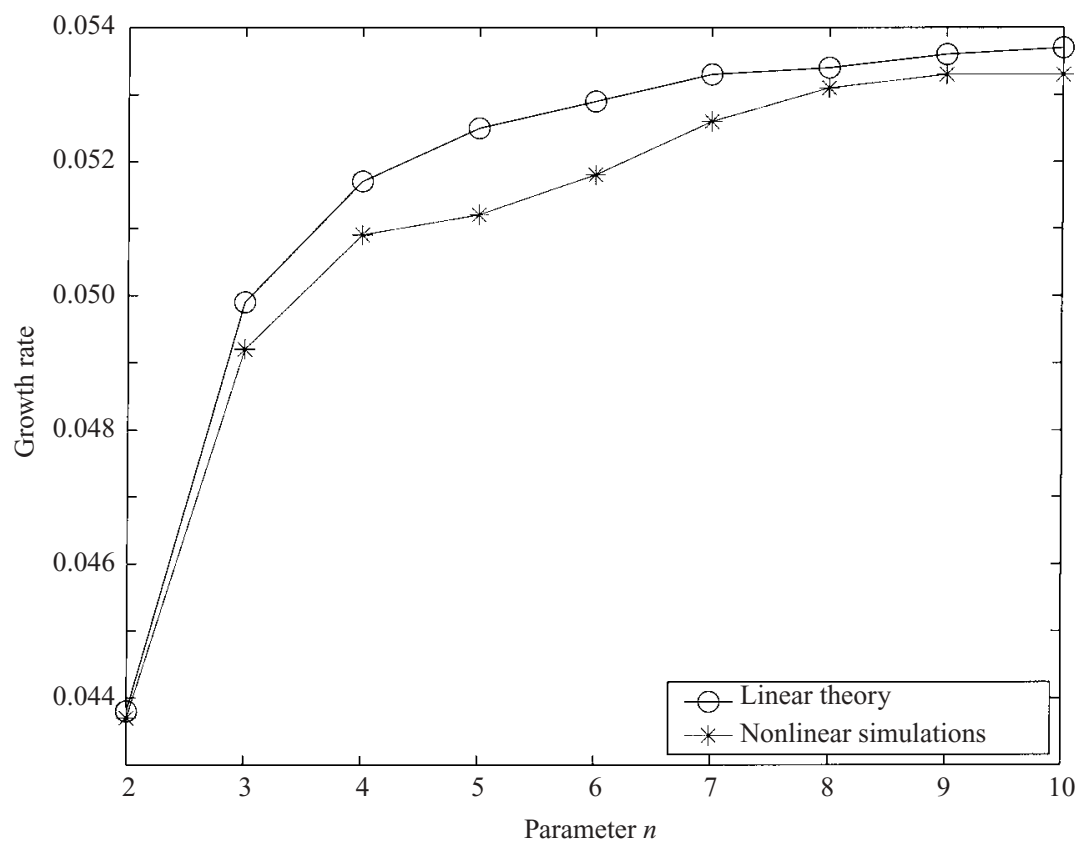

FIGURE 10. A comparison of the growth rates calculated from the linear theory with those obtained from the nonlinear simulations with $\omega=\pi / 4, \delta=1 / 2, \epsilon=1 / 2$ and $q_{0}=1$.
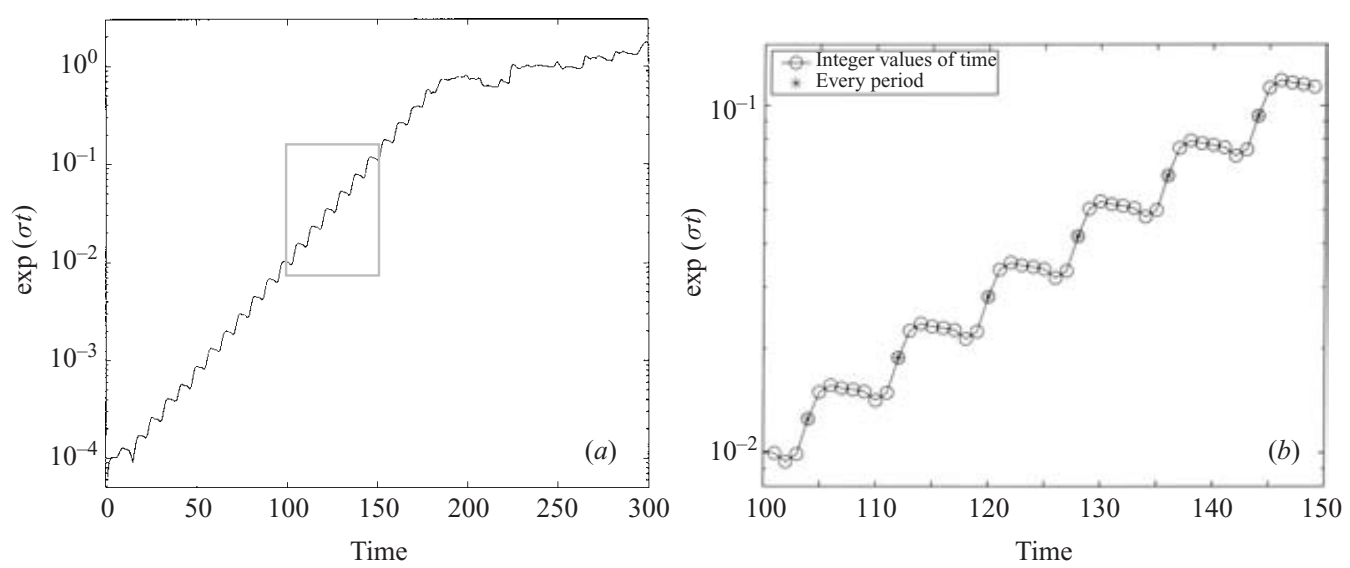

FIgURE 11. A plot of the amplitude as a function of time for the case of $n=4 ;(b)$ is a magnification of the boxed region in $(a)$. Observe the interval in time where the envelope of the curve is linear, meaning that the growth is exponential. Plot $(b)$ shows that during one period, the amount of growth is asymmetric with respect to the amount of decay.

the curve grows linearly, signifying exponential growth. Note that the increase is not monotonic, as needed be for steady instabilities, but rather the growth after every period is monotonic. Throughout each period of linear growth there are intervals of growth and decay, the former being shorter but steeper than the latter. The asterisks in figure 11(b) mark the regions of growth. 

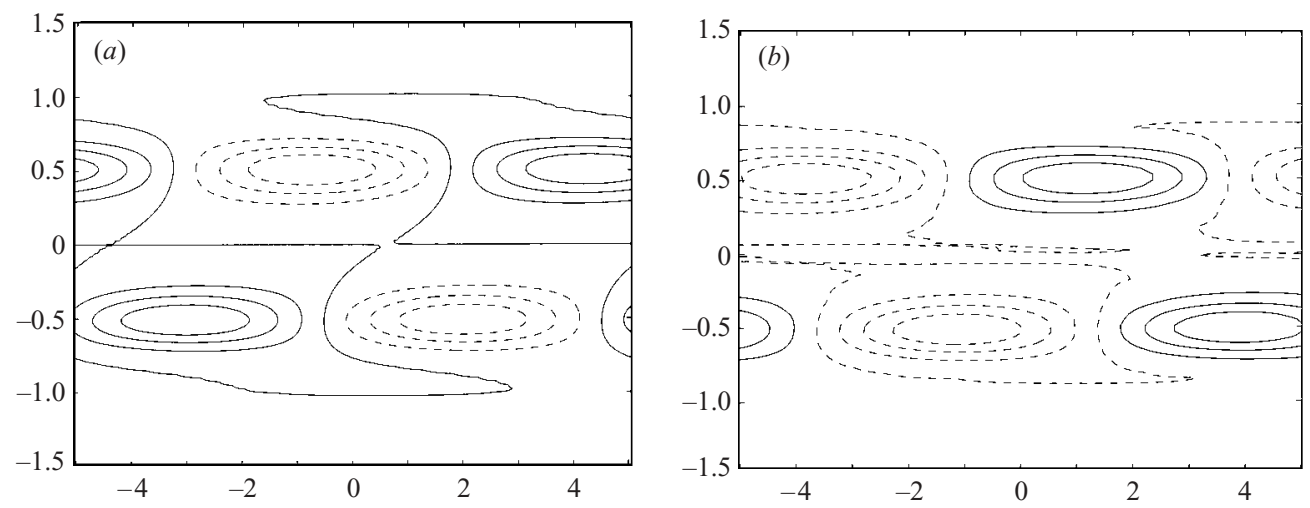

FIGURE 12. A comparison of the structures that grow due to linear instability for $n=2$ : (a) linearly unstable mode for $n=2 ;(b)$ nonlinear structure for $n=2$ at $t=104.0$.

The structures of the growing instabilities for $n=2$ are illustrated in figure 12; $(a)$ and $(b)$ are obtained from the linear and nonlinear calculations, respectively. The size and shape of the structures are very similar. This, coupled with the strong quantitative similarity in the growth rates computed from linear and nonlinear calculations, signifies that the linear theory is indeed accurate in predicting the onset of instability. We remark that the similarity worsens with increasing $n$, because the structures decrease in size and the grid cannot resolve them as well.

In all of these instabilities, there are two lines of alternating elliptical vortices that appear, centred at $y= \pm 0.5$, where the vorticity of the oscillatory state is steepest. The aspect ratios of the minor and major axes are $O\left(10^{-2}\right)$ which gives eccentricities close to 1 . We note that the width of these eddies tends to shrink with increasing $n$. During the stage of linear growth the eddies centred at $y= \pm 0.5$ travel in the negative and positive $x$-directions, respectively, at constant and equal phase speeds. The phase speeds are such that each vortex returns to its original position after two periods, i.e. $c=\pi /(k T)$. This indicates that this instability is indeed subharmonic.

The results from one particular simulation with $n=4$, at a resolution of $256 \times 256$, can be found at the web site http://puddle.mit.edu/ glenn/fjpoulin/ which contains simulations of the perturbation vorticity and total vorticity in gif and mpeg format (P8n4k067perturb.gif, P8n4k067perturb.mpg, P8n4k067total.gif and P8n4k067total.mpg). Figure 13 shows ten snapshots of the total vorticity as the basic state becomes parametrically unstable and then turbulent. In the simulations, the perturbations continue to grow until they become order-one quantities, and they then cease to increase in amplitude. The structures that develop are complicated, but we describe some qualitative features that arise. In all cases, the motion is chaotic and generates turbulence. The vortices extend further out laterally, essentially creating a mixing region that is larger than the width of the jet. We also observe that the cyclones tend to be larger and stronger and circular. Indeed, the vorticity of these eddies is larger than 1; the instability has brought some of the exterior fluid down into the forcing region during the phase when the forcing is causing the vorticity to increase. This fluid moves out before the forcing reverses and thereby retains its elevated vorticity. At the end of the experiment, the peak vorticity is 1.98; the minimum value, -1.02 , is less than zero for similar reasons. The anticyclones, in contrast, are generally thinner, more stretched and filament-like. Presumably, the cyclones are stronger due 

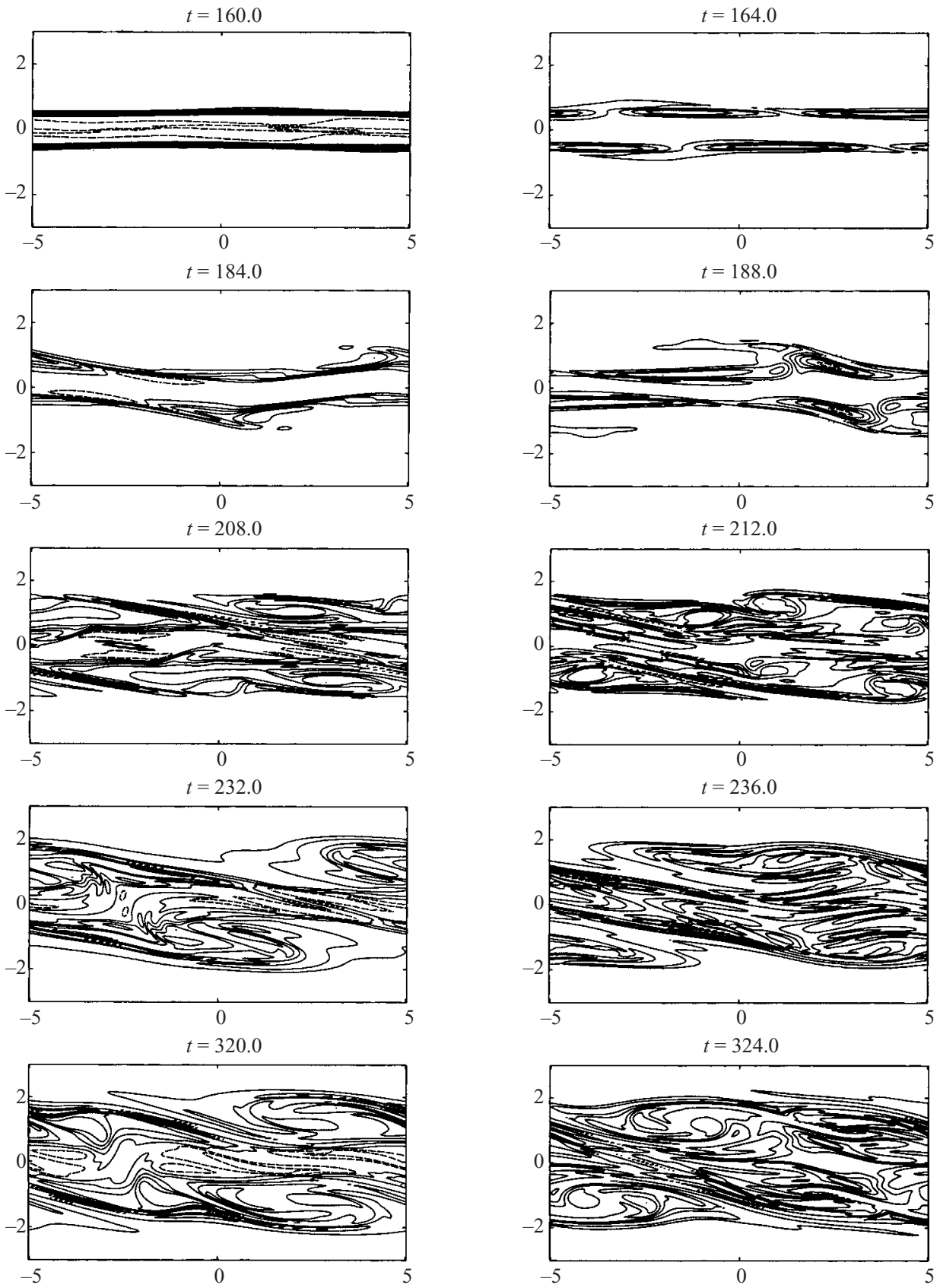

FIGURE 13. Snapshots of the total vorticity in the turbulent state where the period is $T=8$. The solid and dashed lines denote positive and negative contours, respectively.

to the fact that the background shear is cyclonic. We also note that the phase speeds of the vortices are precisely those predicted from the linear stability analysis. There appears to be a symmetry between the two sides of the jet that is composed of a $180^{\circ}$ rotation and an along-flow translation. However turbulent the flow becomes, this symmetry always appears to be maintained. 


\section{Summary and discussion}

Studies of parametric instability in geophysical fluid dynamics have typically focused on its relevance to gravity waves. There has been little investigation of how they can arise in oscillatory shear flow. Some of the most notable exceptions are Greenspan \& Benney (1963), Kelly (1965, 1967), Rosenblat (1968) and Pedlosky \& Thomson (2002). The particular physical problem we address had not yet been studied: it is the interaction of a steady flow with an oscillatory parallel flow in the two-dimensional vorticity equation without any contracting layers. Most of these other studies have been limited to linear theory, whereas our analysis goes beyond this and illustrates the nonlinear evolution of the instability mechanism.

We believe that our work and that of Pedlosky \& Thomson (2002) are the most relevant to atmospheric and oceanic instabilities. Their study differs from and is complementary to the present study in several significant ways. They consider the role of time-dependent mean shear flows in the baroclinic instability problem so that the fundamental nature of the instability itself differs. However, in addition, the focus of their study is the behaviour of the weakly nonlinear dynamics as affected by the timedependent shear. This necessitates a restriction to a region in parameter space near the neutral curve of the classical, steady problem and hence a small alteration of shear as a function of time. Attention in that study is centred on explaining the qualitatively different behaviour (e.g. steady, periodic or aperiodic) occurring depending on whether the time-mean flow is stable or unstable, and, in the former case, whether the instantaneous flow (the 'snapshot') is stable. Instead, here we focus attention on barotropic velocity profiles of both the broken line and continuous type and examine the linear problem in detail. We present some strongly nonlinear numerical solutions that describe the fate of strongly unstable modes of parametric instability.

In our linear analysis, we have determined that all pure oscillatory shear flows of zero temporal mean are transiently stable. When the mean is non-zero, the oscillatory flows are unstable for exactly the same wavenumbers that are unstable according to the steady theory. The linear theory for the two-contour model with a background steady shear produced parametric instability tongues in parameter space similar to those in Mathieu's equation. This instability implies that otherwise stable (unstable) flows can be destabilized (stabilized) by the oscillations. The multiple-scales method yielded analytical expressions for the growth rate of the first subharmonic which agreed with the direct solution of the linear stability problem. The tendency is for the growth rate of the instability to increase with increasing background shear, but to decrease with increasing wavenumbers and frequencies. We determined that the barotropic instabilities that are present in the steady case tend to be damped and moved to larger wavelengths as a result of the oscillations. Also, regardless of whether the basic state is stable or unstable at every instant in time, the strength and structure of the parametric instabilities are very similar.

Finally, we delved into the more physical problem of oscillatory continuous basic states. The linear stability problem illustrates that parametric resonance does occur for a large class of oscillatory shear flows that are Gaussian-like. We observed that shear flows must be sufficiently steep to generate this type of instability and the two-contour problem of $\S 4$ produces the largest growth rates. We also studied the nonlinear evolution of some of these unstable states and found a strong similarity between the growth rates and structures predicted from linear theory and those calculated from the nonlinear simulations. The vortices produced are stretched and elliptical in shape, with widths that decrease with increasing basic-state steepness. After the linear growth ceases, the unstable region becomes wider than the jet and 
generates turbulence. This mixes fluid around the boundary of the jet but not across the jet.

This article and Pedlosky \& Thomson (2002) are the first works to explore the importance of parametric resonance in the context of horizontal and vertical shears, respectively, in time-dependent shears for large-scale geophysical flows. Given that a theoretical foundation has now been set, it is of great interest to study geophysical oscillatory flows such as the Semi-Annual Oscillation (Shepherd 2000), the QuasiBiennial Oscillation (Baldwin et al. 2001), and the Georges Bank (K. H. Brinks, personal communication) and Cape Cod Bay Oscillation (Poulin 2002) in order to observe the parametric modes and how they arise in nature.

The importance of this work spreads beyond periodically forced systems since amplitude vacillations can be generated in otherwise steady flows through nonlinear dynamics (Pedlosky 1987). Kelly (1967) studied the case in which oscillations from the primary instability can interact with the basic state to generate a secondary instability that is even stronger. However, this was done for only one particular profile. Indeed, there is a large range of problems in which the primary instability can interact with the mean flow in order to destabilize or perhaps stabilize the flow. This is an important issue in the transition to turbulence that requires further investigation.

This work also shows the possible incompleteness of using averaged profiles in geophysical situations as the basis for parameterizations for eddy fluxes of heat and momentum. There may certainly be instances when averaging yields meaningful results; however, there must also be regimes where they fail since they cannot account for parametric instability. It is of great significance to try to determine in what regimes we cannot expect parameterizations to yield meaningful results.

The authors would like to thank the reviewers and the editor for their constructive comments. F.P.'s and G.F.'s research was supported by grants from NSF, OPP9910052 and OCE-0137023. J.P.'s research is supported in part by a grant from NSF, OCE-9901654.

\section{REFERENCES}

Baldwin, M., Gray, L., Dunkerton, T., Hamilton, K., Haynes, P., Randel, W., Holton, J., Alexander, M., Hirota, I., Horinouchi, T., Jones, D., Kinnersley, J., Marquardt, C., Sato, K. \& Takahashi, M. 2001 The Quasi-Biennial Oscillation. Rev. Geophys. 39, 179-229.

Benjamin, T. \& Ursell, F. 1954 The stability of the plane free surface of a liquid in vertical periodic motion. Proc. R. Soc. Lond. A 225, 505-515.

Broutman, D., Macaskill, C. \& McIntyre, M. 1997 On Doppler-spreading models of internal waves. Geophys. Res. Lett. 24, 2713-2816.

CASE, K. 1960 Stability of inviscid plane Couette flow. Phys. Fluids 3, 143-154.

Davis, S. 1976 The stability of time-periodic flows. Annu. Rev. Fluid Mech. 8, 57-74.

Drazin, P. \& ReID, W. 1995 Hydrodynamic Stability. Cambridge University Press.

DritsChel, D. 1989 On the stabilization of a two-dimensional vortex strip by adverse shear. J. Fluid. Mech. 206, 193-221.

ERN, P. \& WeSFreID, J. 1999 Flow between time-periodically co-rotating cylinders. J. Fluid Mech. 397, 73-98.

Fletcher, C. 1991 Computational Techniques for Fluid Dynamics. Springer.

Greenspan, H. \& Benney, D. 1963 One shear-layer instability, breakdown and transition. J. Fluid Mech. 15, 133-153.

Hu, H.-C. 1995 Effect of a time-periodic axial shear flow upon the onset of taylor vortices. Phys. Rev. E 51, 3242-3251.

Kelly, R. 1965 The stability of an unsteady Kelvin-Helmholtz flow. J. Fluid Mech. 22, 547-560. 
KelLy, R. 1967 On the stability of an inviscid shear layer which is periodic in space and time. J. Fluid Mech. 27, 656-689.

KerczeK, C. V. 1982 The instability of oscillatory plane poiseuille flow. J. Fluid Mech. 116, 91-114. Lopez, J. \& Marques, F. 2002 Modulated Taylor-Couette flow: Onset of spiral modes. Theor. Comput. Fluid Dyn. 16, 59-69.

Marques, F. \& LopeZ, J. 1997 Taylor-Couette flow with axial oscillations of the inner cyliner: Floquet analysis of the basic flow. J. Fluid Mech. 348, 153-175.

Marques, F. \& LoPEZ, J. 2000 Spatial and temporal resonances in a periodically forced hydrodynamic system. Physica D 136, 340-352.

McEwan, A. \& Robinson, R. 1975 Parametric-instability of internal gravity-waves. J. Fluid Mech. 67, 667-687.

Meseguer, A. \& Marques, F. 2000 On the competition between centrifugal and shear instability in spiral Couette flow. J. Fluid Mech. 402, 33-56.

Normand, C. 2000 Stability of time-periodic flows in a Taylor-Couette geometry. In Physics of Rotating Fluids: Selected Topics of the 11th Intl Taylor-Couette Workshops. Lecture Notes in Physics, vol. 549 (ed. C. Egbers \& G. Pfister), pp. 67-83, Springer.

Pedlosky, J. 1987 Geophysical Fluid Dynamics. Springer.

Pedlosky, J. \& Thomson, J. 2002 Baroclinic instability of time-dependent currents. J. Fluid Mech. (submitted).

Poulin, F. 2002 The instability of time-dependent jets. PhD thesis, MIT, Department of Mathematics.

Pullin, D. 1992 Contour dynamics methods. Annu. Rev. Fluid Mech. 24, 89-115.

Rosenblat, S. 1968 Centrifugal instability of time-dependent flows. Part 1. Inviscid, period flows. J. Fluid Mech. 33, 321-336.

Shepherd, T. 2000 The middle atmosphere. J. Atmos. Sol.-Terres. Phys. 62, 1587-1601.

Silveira, I. D. \& Flierl, G. 2002 Eddy formation in 2 1/2-layer, quasigeostrophic jets. J. Phys. Oceangr. 32, 729-745.

Staquet, C. \& Sommeria, J. 2002 Internal gravity waves: From instabilities to turbulence. Annu. Rev. Fluid Mech. 34, 559-593.

STOKER, J. 1950 Nonlinear Vibrations in Mechanical and Electrical Systems. Interscience.

WALSH, T. \& DonNELLY, R. 1988 Taylor-Couette flow with periodically corotated and counterrotated cylinders. Phys. Rev. Lett. 60, 700-703.

ZABUSKY, N. \& Overman, E. 1983 Regularization of contour dynamical algorithms, I. tangential regularization. J. Comput. Phys. 52, 351-373. 